\title{
Optical Scanner for 3D Radiotherapy Polymer Gel Dosimetry
}

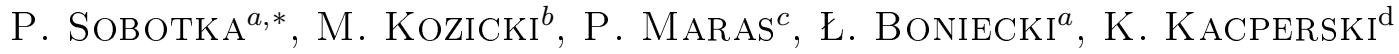 \\ AND A.W. DOMAŃSKI ${ }^{a}$ \\ ${ }^{a}$ Faculty of Physics, Warsaw University of Technology, Koszykowa 75, 00-662 Warsaw, Poland \\ ${ }^{b}$ Department of Man-Made Fibres and European Centre of Bio- and Nano-Technology (ECBNT) \\ Łódź University of Technology, S. Żeromskiego 116, 90-924 Łódź, Poland \\ ${ }^{c}$ Teletherapy Department, Copernicus Hospital, Pabianicka 62, 93-513 Łódź, Poland \\ ${ }^{\mathrm{d}}$ Maria Skłodowska-Curie Memorial Cancer Center and Institute of Oncology \\ Department of Medical Physics, W.K. Roentgena 5, 02-781 Warsaw, Poland
}

\begin{abstract}
Sophisticated techniques employed in radiotherapy for irradiation of tumours require comprehensive dosimetry allowing for precise, high resolution measurements of radiation dose distribution in three dimensions and verification of treatment planning systems. Polymer gel dosimetry has been shown to be a unique technique for such the purpose. If exposed to ionizing radiation, radical polymerisation and crosslinking of monomeric components take place in a 3D polymer gel dosimeter, leading to the formation of large polymeric structures that scatter visible light. This feature allows for optical observation of the effects of the absorbed dose and its distribution. Presently, magnetic resonance imaging is employed most often for the analysis of the 3D polymer gel dosimeters. However, much attention is also being given to the development of optical computed tomography since this technique is hoped to serve as a substitute for expensive and not easily available magnetic resonance imaging. The optical scanner presented in this work consists of a laser diode, a scanning system and a signal detector. A 3D polymer gel dosimeter is measured in an immersion liquid in order to reduce deflection of the light from the dosimeter phantom. The very first results were obtained with the newly constructed scanner and PABIG ${ }^{\mathrm{nx}} 3 \mathrm{D}$ polymer gel dosimeter, which was inhomogeneously irradiated with ${ }^{192}$ Ir brachytherapy source. The results have been contrasted with those for the magnetic resonance imaging and are presented in this work together with the description of the developed instrument. Currently, the optimization of the optical scanner is performed.
\end{abstract}

PACS: 87.50.Gi, 42.66.Si, 87.53.Bn

\section{Introduction}

Radiotherapy of tumours requires state-of-the-art dosimetry allowing for 3D high resolution measurements of ionising radiation dose distribution. Verification of treatment planning systems (TPS) and TPS-generated plans of tumours irradiation should also be possible with the dosimetry. Currently, the only entirely 3D dosimetry available is the polymer gel dosimetry employing $3 \mathrm{D}$ polymer gel dosimeters, which serve as soft tissue and water equivalent phantoms and allow for the recording of integrated absorbed dose distributions in three dimensions in clinical radiation therapy. Recently, a proposition of yet another $3 \mathrm{D}$ radiation detector has attracted attention as well [1].

Historically, many gel dosimeters have been proposed including those that are based on the Fricke dosimeter and the conversion reaction of $\mathrm{Fe}^{2+}$ to $\mathrm{Fe}^{3+}$ shown elsewhere [2-9]. The advancement in these dosimeters

\footnotetext{
* corresponding author; e-mail: sobotka@if.pw.edu.pl
}

was a substitution of the reactive ferrous substrate with vinyl monomers [10-12] to arrive at the polymer gel dosimeters. 3D polymer gel dosimeters are physical hydrogels containing radiation-sensitive vinyl compounds. Upon ionising radiation, the compounds undergo polymerisation and cross-linking which manifest in the appearance of opacity. The formula of these dosimeters was altered in order to facilitate the preparation procedures which have led to so-called normoxic polymer gel dosimeters [13]. Afterwards, many new formulae have appeared; for instance, MAGAT and PAGAS [14, 15], MAGAS [16] and PAGAT [17]. Among polymer gel dosimeters, those which are based on $N$-vinylpyrrolidone (VIPAR) and poly(ethylene glycol)diacrylate (PABIG; the acronyms stem from the compositions) have been of interest. The initially proposed VIPAR and PABIG were customized to better serve $3 \mathrm{D}$ dosimetry. The formula of VIPAR [18, 19] was changed to $\operatorname{VIPAR}^{\mathrm{d}}$ by increasing the concentration of $N$-vinylpyrrolidone and gelatine. This lowered the dose threshold [20]. Afterwards, VIPAR $^{\mathrm{d}}$ was changed by the addition of substances eliminating the oxygen inhibition effect of radiation induced 
polymerisation and crosslinking. The acronym VIPAR ${ }^{\text {nd }}$ was used [21], which has later been abbreviated to VIP $[22,23]$. Another modification of the VIPAR polymer gel has also been proposed elsewhere [24]. The PABIG polymer gel dosimeter [25, 26] was examined for radiotherapy dosimetry [26]. It has been recently changed by the addition of substances typical for normoxic type compositions [23].

The features of polymer gel dosimeters allow for use of such techniques as X-ray computed tomography [21, 22], ultrasonography [23] and most often magnetic resonance imaging $[2,3,12]$ for quantitative analysis of the dosimeters after irradiation. Much attention is also being given to the optical computed tomography $[18,19]$. This technique, which is under development for 3D dosimetry, is hoped to solve in the future the problem of limited access to the modern magnetic resonance imaging equipped with a multi-echo sequence option, which was also reported elsewhere [21].

In this work, the authors' first results on the construction and application of an optical scanner for polymer gel dosimetry are presented. A method of 3D scanning with the aid of an optical scanner requires that several conditions are fulfilled in order to be a respected one. These include that the method allows for the $3 \mathrm{D}$ scanning in short time relative to the magnetic resonance imaging on the condition that the resolution is similar to that of the magnetic resonance imaging. Besides, the method should allow for 3D reconstruction of signal distribution and further transformation into 3D absorbed dose distribution after application of a calibration equation. The optical scanning method should be coupled with appropriate software allowing for comparison of 3D dose distribution measured with that calculated with a TPS or measured with other dosimeters used in medical radiation dosimetry. These are currently of interest for the authors of this work.

\section{Materials and methods}

\subsection{Preparation of $3 D$ polymer gel dosimeter}

The method of the dosimeter preparation was analogous to the one described elsewhere [27]. Poly(ethylene glycol)diacrylate (PEGDA, Mn $=700 \mathrm{~g} / \mathrm{mol}$; Aldrich), $N, N^{\prime}$-methylenebisacrylamide (MBA; Aldrich), gelatine (type A, 300 Bloom; Sigma), copper sulphate pentahydrate $\left(\mathrm{CuSO}_{4} \times 5 \mathrm{H}_{2} \mathrm{O}\right.$; Chempur), ascorbic acid (AsAc; Chempur) and distilled water were used for the preparation of $\mathrm{PABIG}^{\mathrm{nx}}$ samples. MBA $(4 \% \mathrm{w} / \mathrm{v})$ was dissolved at $45^{\circ} \mathrm{C}$ and next gelatine $(5 \% \mathrm{w} / \mathrm{v})$ was added and the mixture was stirred until it was transparent. Then, it was cooled down to around $25^{\circ} \mathrm{C}$ and PEGDA $(4 \% \mathrm{w} / \mathrm{v})$ was added. Finally, copper sulphate pentahydrate $(0.0004 \% \mathrm{w} / \mathrm{v})$ and ascorbic acid $(0.007 \% \mathrm{w} / \mathrm{v})$ were mixed with the solution. After careful mixing, the liquid composition was poured into brachytherapy vial (height: $150 \mathrm{~mm}$; inside diameter: $80 \mathrm{~mm}$; Fig. 1) and left for $24 \mathrm{~h}$ at $23^{\circ} \mathrm{C}$ in order to solidify.

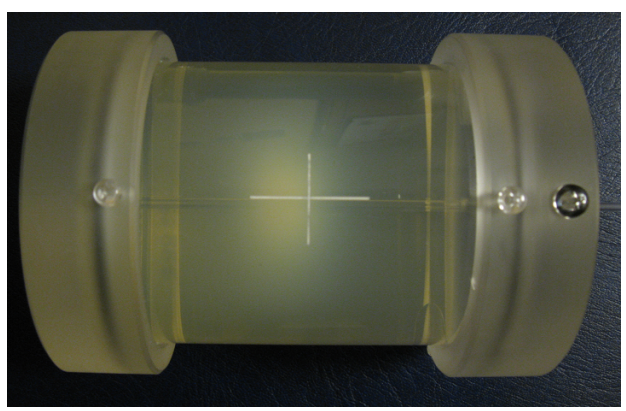

Fig. 1. PABIG ${ }^{\mathrm{nx}}$ polymer gel dosimeter after irradiation with ${ }^{192} \mathrm{Ir}$ brachytherapy source (conditions: $10 \mathrm{~Gy}$ at $10 \mathrm{~mm}$ distance from the source). The opaque region in the middle of the sample corresponds to the polymerisation and crosslinking of the monomeric components which occurred after irradiation.

\subsection{Irradiation with ${ }^{192}$ Ir}

A brachytherapy vial of PABIG ${ }^{\text {nx }}$ was irradiated with a ${ }^{192}$ Ir source (303.2 GBq; 8.2 Ci; RAKR 33.3 mGy/h; capsule dimensions: $1.1 \mathrm{~mm}$ diameter; $5.1 \mathrm{~mm}$ length; source pellet dimensions: $0.6 \mathrm{~mm}$ diameter; $3.5 \mathrm{~mm}$ length; GammaMed 12i HDR, Varian Medical Systems, USA). The source was placed automatically inside the dosimeter for the time required in order to deliver $10 \mathrm{~Gy}$ at a distance of $10 \mathrm{~mm}$ from the source, perpendicular to the longer axis of the source at a single dwell position. The vial after irradiation is presented in Fig. 1.

\subsection{Magnetic resonance imaging}

The measurements of PABIG ${ }^{\mathrm{nx}}$ vial was performed with the aid of a Siemens Avanto magnetic resonance whole body scanner (1.5 T; syngo MR B17 software). An $\mathrm{RF}$ head coil was used for the PABIG ${ }^{\mathrm{nx}}$ vial. Before scanning, the sample was kept in an air conditioned room with the MRI device. Any transfer of the gel dosimeter after preparation, irradiation or during MR scanning was done with the aid of isothermal bag. A 16-equidistant-echo multi-slice sequence was optimised with respect to SNR, bearing in mind a desired resolution of scanning and limited time for the measurement. Namely, TR equalled $4040 \mathrm{~ms}$; the first echo was $50 \mathrm{~ms}$ and the last one was $800 \mathrm{~ms}$. The vial was scanned so that only the whole irradiated region was covered. $2 \mathrm{D}$ planes were received perpendicularly to the catheter's longer axis. The slice thickness was set to $1 \mathrm{~mm}$ and the in-plane resolution was $1.02 \times 1.02 \mathrm{~mm}^{2}$.

After MR scanning, 2D MR echo slices were used for calculations of the spin-spin relaxation time $\left(T_{2}\right)$ and corresponding $R_{2}\left(1 / T_{2}\right)$ planes. The data was further processed employing only 14 echoes after discarding the first two following a suggestion published elsewhere [20]. Afterwards, the calculations of profiles and dose distribution was done with the aid of polyGeVero ${ }^{\circledR}$ software (see Sect. 2.4). 


\subsection{MRI data processing}

All calculations of MRI data was done with new software, polyGeVero ${ }^{\circledR}$, which succeeded GeVero ${ }^{\circledR}$ software $[21,22]$. The software allows for number operations in four workspaces. The calibration workspace is equipped with tools allowing for calculations of calibration equations. In the case of the ${ }^{192} \mathrm{Ir}$ irradiated sample the calculations were based on the line approximation approach [23]. The resulting calibration equations can be stored in a database together with the full characteristics of experiments and polymer gel dosimeters. Another workspace is used for dose distributions calculation. Afterwards, all other operations aiming at a comparison of a gel dosimetry dose distribution with a dose distribution received with the aid of a treatment planning system can be performed in the gel dosimetry vs. TPS workspace. There are several tools available for the users that are typical for the medical dosimetry in radiotherapy. The software was tested for accuracy. Further details will be announced in a separate publication.

\subsection{Optical scanner and imaging algorithm}

The optical scanner consists of a light source, a scanning system controlled by software, a light detector and a system that reads the data from the detector [22] (Fig. 2). The PABIG $^{\mathrm{nx}}$ polymer gel dosimeter vial was placed in a tank made of poly(methyl methacrylate) (dimensions $21 \times 17 \times 17 \mathrm{~cm}^{3}$ ) with an immersion liquid (water) to reduce deflection of the laser beam from the dosimeter vial (Fig. 2). The scanning system includes translating and rotating stage, which moves a tank with the $\mathrm{PABIG}^{\mathrm{nx}}$ vial perpendicular to the laser beam which rotates PABIG $^{\text {nx }}$ vial according to the translation scanning procedure for each angular position of $\mathrm{PABIG}^{\mathrm{nx}}$ vial. As a light source a red semiconductor laser diode is used (632 nm; TECHNO LASER, Poland), whereas as a detector a silicon photodiode $37 \mathrm{~S}$ is applied (Institute of Electron Technology, Poland) which is dedicated to radiation of the wavelength range of $550-650 \mathrm{~nm}$. The diameter of detector is $25 \mathrm{~mm}$ and the active area is $1 \mathrm{~cm}^{2}$. The signal in form of transmitted light through the tank with the dosimeter is collected with the detector by means of 10 bit $\mathrm{AC} / \mathrm{DC}$ converter with a frequency of $20 \mathrm{~Hz}$ (AUTOSTER, Poland) and is saved to a file.

The system of operation of the optical scanner is based on the intensity measurement of the light beam passing through the $\mathrm{PABIG}^{\mathrm{nx}}$ vial in the tank by use of the photodetector. The intensity is inversely proportional to the opacity in the $\mathrm{PABIG}^{\mathrm{nx}}$ that appears after irradiation (radiation dose $\sim$ opacity).

In this study, the intensity of a laser beam passing along the transversal plane perpendicular to the longer axis of the $\mathrm{PABIG}^{\mathrm{nx}}$ vial and crossing the source position was measured at 531 equidistant points. The measurement was repeated for 360 rotation angles, in $1^{\circ}$ steps, of the entire tank, giving the complete set of tomographic projections for the single transversal plane. It was assumed that the light attenuation is described by the

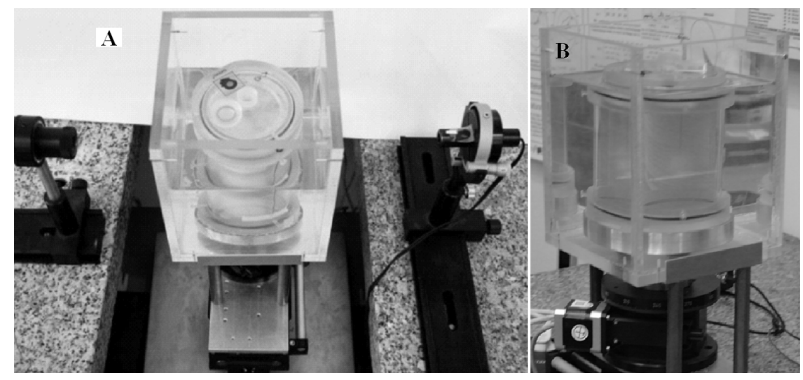

Fig. 2. Components of the optical scanning system for measurements of the 3D polymer gel dosimeters. (A) The measurement units and a tank with an immersion liquid and the PABIG ${ }^{\mathrm{nx}}$ vial inside $\left({ }^{192} \mathrm{Ir}\right.$ irradiated); (B) a side view of the $\mathrm{PABIG}^{\mathrm{nx}}$ vial in the tank.

Lambert-Beer exponential law and therefore the image of the absorption coefficient can be reconstructed by taking the logarithm of the measured data and applying the standard filtered back projection (FBP) algorithm, similarly as in the case of X-ray computed tomography [23]. No additional low pass filtering was applied. The software used for control of the optical scanner allows changing the width of the scan depending on a diameter of a dosimeter gel phantom.

\section{Results and discussion}

\subsection{MRI scanning}

Magnetic resonance imaging delivered information on spin-spin $T_{2}$ relaxation time distribution in the ${ }^{192} \mathrm{Ir}$ irradiated $\mathrm{PABIG}^{\mathrm{nx}}$ polymer gel dosimeter with high resolution in three-dimensions. This was further transformed to $R_{2}$ relaxation rate distribution $\left(R_{2}=1 / T_{2}\right)$ and the calibration equation was derived with polyGeVero ${ }^{\circledR}$ software through the comparison of $R_{2}$ vs. distance from the centre of the active part of ${ }^{192} \mathrm{Ir}$ across the longer axis of the source with the dose vs. distance relation calculated for this source after application of the line approximation algorithm (see Sect. 2.2). The following equations were obtained: $R_{2}=0.0787 D+1.1354(1-16 \mathrm{~Gy}$; quasi linear dose response; $\left.R^{2}=0.99\right)$ and $R_{2}=1.1315+0.0873 D-$ $8.5226 \times 10^{-4} D^{2}+3.8934 \times 10^{-6} D^{3}-5.7073 \times 10^{-8} D^{4}$ $(0.5-40 \mathrm{~Gy})$. It was concluded that the PABIG ${ }^{\mathrm{nx}}$ dosimeter saturates after absorption of a dose of over 40 Gy. Therefore, it finds application to radiotherapy dosimetry, since typical treatment radiation dose is 60 Gy delivered in 2 Gy fractions.

The polynomial calibration equation was applied for the calculation of $3 \mathrm{D}$ dose distribution in $\mathrm{PABIG}^{\mathrm{nx}}$ and the result is presented in Fig. 3. It was concluded that the dose distribution from ${ }^{192}$ Ir in this dosimeter is characterised by typical apple-like shape and resembles the one reported elsewhere for another polymer gel dosimeter [24]. For the purpose of this work, a single plane perpendicular to the longer axis of the PABIG $^{\text {nx }}$ vial and 
passing through the centre of the source's active part was selected (Fig. 4A) and a relative dose distribution was calculated. Afterwards, a profile across the plane middle part was obtained and is presented in Fig. 4B. It can be seen that the dose distribution along the transverse bisector of the ${ }^{192} \mathrm{Ir}$ is very similar.

The absorbed dose by the $\mathrm{PABIG}^{\mathrm{nx}}$ polymer gel dosimeter increases when the distance from the source decreases. However, at the distance lower than $2.5 \mathrm{~mm}$ from the source, the absorbed dose measured by $\mathrm{PABIG}^{\mathrm{nx}}$ drops down. The reason for this behaviour is the measurable dose range by $\mathrm{PABIG}^{\mathrm{nx}}$, which was described above to be up to $40 \mathrm{~Gy}$. At the very close vicinity of the high dose rate iridium isotope the absorbed dose may rise to hundreds of Gy. For instance, at similar irradiation conditions the absorbed dose by VIPAR polymer gel dosimeter may reach over $300 \mathrm{~Gy}$ at around $1.5 \mathrm{~mm}$ distance from the source $[24,25]$. Such the high absorbed dose may induce processes of degradation of the $\mathrm{PABIG}^{\mathrm{nx}}$ polymer structure after the monomeric components complete conversion to crosslinked structures at lower absorbed doses [26]. This in turn can result in the formation of the lower molecular weight products causing an increase in $T_{2}$ values (and decrease in $R_{2}$ ), which reflects in the lower calculated absorbed dose at the vicinity of the ${ }^{192} \mathrm{Ir}$. Some influence of the artefacts at the close distance from the catheter $(\approx 1.5 \mathrm{~mm}$ from the catheter's wall) that appears for the magnetic resonance imaging might add to this decrease observed in the relative dose distribution. The graphs presented in Fig. 4 were used for further discussion of the $\mathrm{PABIG}^{\mathrm{nx}}$ optical scanning results.

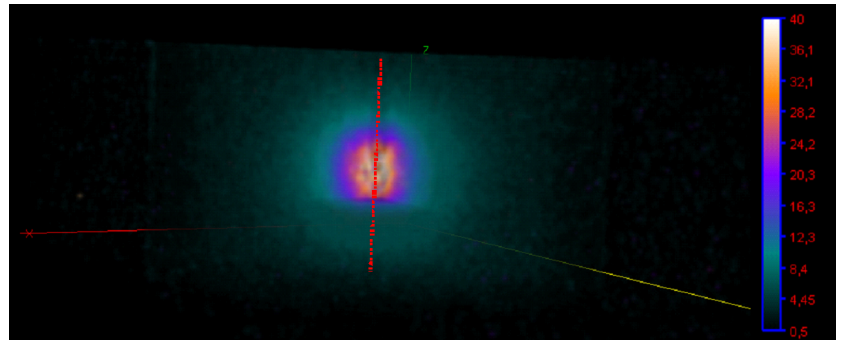

Fig. 3. 3D dose distribution in $\mathrm{PABIG}^{\mathrm{nx}}$ polymer gel dosimeter after irradiation with brachytherapy source, ${ }^{192} \mathrm{Ir}$, placed centrally inside the dosimeter as described in Sect. 2.2. The scale on the left represents the absorbed dose range of $0.5-40$ Gy. The catheter position through which ${ }^{192}$ Ir was introduced in $\mathrm{PABIG}^{\mathrm{nx}}$ is indicated with a red dot line. The source position was roughly in the middle of this line.

\subsection{Optical scanning}

The first measurement carried out was the one for the unirradiated area of the PABIG ${ }^{\mathrm{nx}}$ polymer gel dosimeter. The result is shown in Fig. 5. This measurement was performed to determine if the curvature of the phantom distorts the measurement. In Fig. 5B the profile
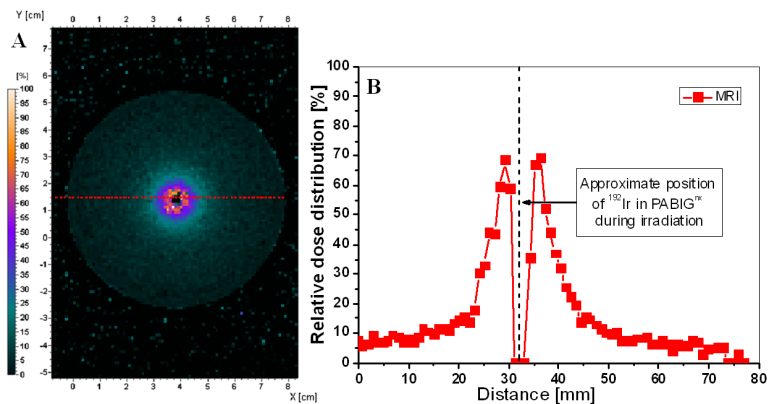

Fig. 4. (A) Reconstruction of a single plane as relative dose distribution across the longer axis of the source and crossing it at the centre of an active part. (B) A profile obtained along $X$ axis centrally from the plane presented in (A), as is dictated with a red dot line.

across the phantom is shown. The graph represents a flat response of the PABIG ${ }^{\mathrm{nx}}$. The mean signal intensity from the inner part of the $\mathrm{PABIG}^{\mathrm{nx}}$ vial and the corresponding standard deviation is equal to $116.2 \pm 0.6$, which means that the use of the container with the liquid immersion minimizes the measurement artifacts related to the shape of the phantom.
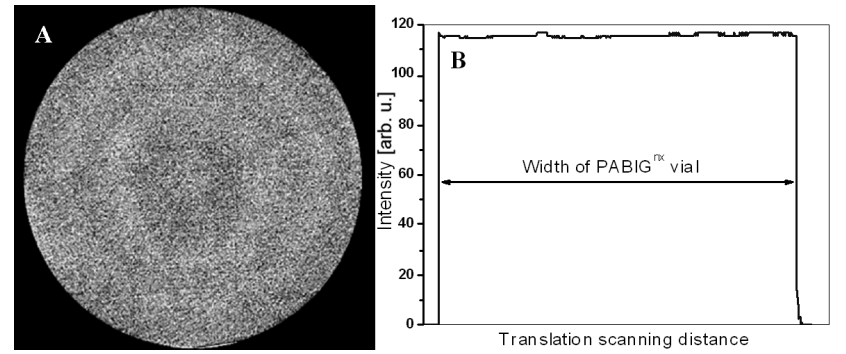

Fig. 5. The measured area of $\mathrm{PABIG}^{\mathrm{nx}}$ that was not irradiated. (A) Reconstruction of a central plane; (B) a profile across the centre of the plane.

The results presented in Fig. 6A and $\mathrm{B}$ correspond to the cross-section of $\mathrm{PABIG}^{\mathrm{nx}}$ in the region of irradiation and maximal absorbed dose and a profile intensity across the middle part of this plane, respectively. The region of irradiation can be easily noticed in the middle part of the plane. The graph shown in Fig. $6 \mathrm{~B}$ presents that the signal intensity decreases when data collection approaches the middle part of the PABIG ${ }^{\mathrm{nx}}$ vial. This is naturally caused by the lower transmission of light due to the higher density of the crosslinked polymer formed in that region of the $\mathrm{PABIG}^{\mathrm{nx}}$ that is at a closer distance from the iridium source. The change of the signal intensity is similar at both sides of the vial's centre, which means that the dose distribution around the ${ }^{192}$ Ir bisector is alike.

The results obtained for the optical scanning allowed for the comparison of profiles obtained from this technique with those of the magnetic resonance imaging. The result is presented in Fig. 7 . It is apparent that the re- 

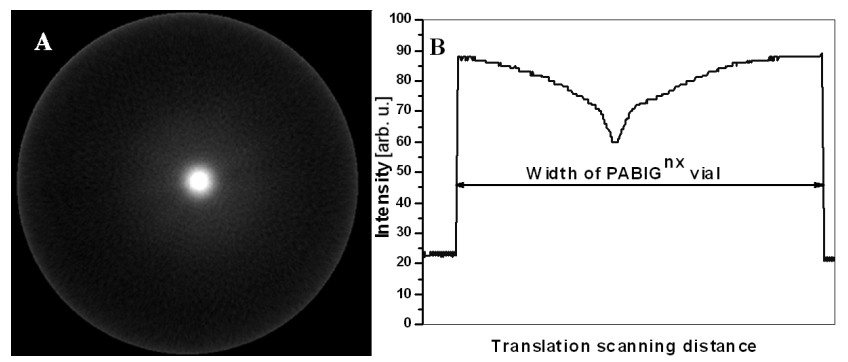

Fig. 6. The measured area of PABIG ${ }^{\text {nx }}$ that was irradiated. (A) Reconstruction of a central plane; (B) a profile across the centre of the plane.

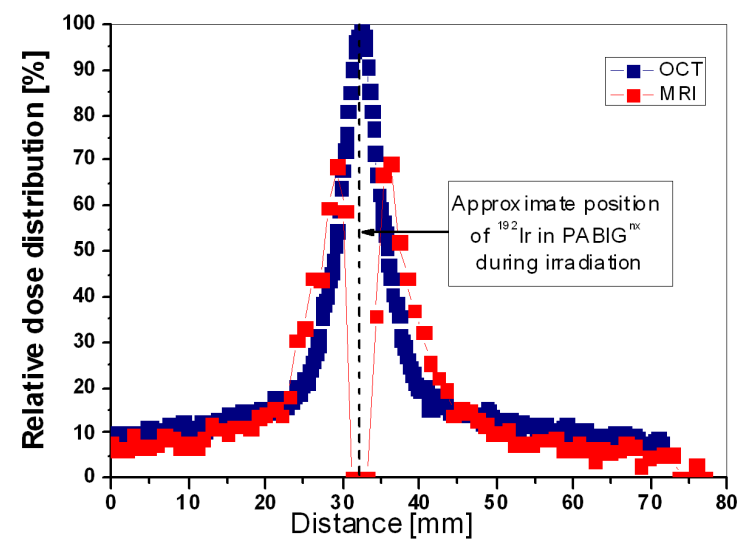

Fig. 7. Comparison of the profiles from the central planes of the irradiated PABIG ${ }^{\text {nx }}$ obtained with the optical scanner (OCT) and the magnetic resonance imaging (MRI).

sults are very similar and promising for the region of the relative dose range $5-70 \%$. Some differences between the data at this dose region may have arisen from the different time of scanning with the optical scanner and the magnetic resonance imaging after the $\mathrm{PABIG}^{\mathrm{nx}}$ irradiation. At first, the dosimeter was scanned with the magnetic resonance imaging and afterwards it was transferred about a month later to be scanned with the other technique. The time gap between the scannings obviously was a reason for some changes occurring in the dosimeter matter due to the post effect and aging phenomena. However, the most important difference between the data is for the region of the relative dose range above $70 \%$. Although this is to be examined in the further studies in detail, we can speculate now that the relative dose measured with the optical scanner is distorted due to very low transmission of light at the close vicinity to the $\mathrm{PABIG}^{\mathrm{nx}}$ vial's centre. In that region, the absorbed dose was the highest and the conversion of the dosimeters components was $100 \%$, which produced the saturated opacity. However, the very middle of the dosimeter's vial was an empty part after a catheter, for which the transmission of light is maximal. This is not visible for the optical scanning data due to the opacity surrounding this part of the dosimeter. Undoubtedly, further studies need to be performed in order to produce calibration curves for the optical scanning and conclude on the dose range possible for measurements with this technique for a $3 \mathrm{D}$ polymer gel dosimeter.

\section{Conclusions}

In this paper the first results are presented for the optical scanner designed and constructed for the polymer gel dosimetry in our group. The first tests of the instrument were performed with the $\mathrm{PABIG}^{\mathrm{nx}} 3 \mathrm{D}$ polymer gel dosimeter which allows for high resolution ionising radiation absorbed dose measurements in radiotherapy dosimetry. The results prove that the proposed optical scanning method allows for the reconstruction of the radiation dose distributions measured via the dosimetry phantom.

Further studies towards the development of this scanning method are required. It is planned to refine and develop the operating software by adding several new features to support the system. It was estimated that the scan time of one layer was more than $2 \mathrm{~min}$ at angular resolution of 1 degree. The whole volume measured may amount to about $12 \mathrm{~h}$ (repeatability of measurement was assessed to be satisfying). Therefore, the measurements are to be speeded up. For this reason several light sources and detectors will be applied. Moreover, we plan focus on the 3D reconstruction of the whole dosimeter's volume measured. After the scanning system is advanced, further studies towards characterisation and its performance should be carried out with some polymer gel dosimeter compositions in order to generate the systems characteristics and procedures of the dosimeters scanning and data processing.

\section{Acknowledgments}

This work was partially financed by the Polish Ministry of Science and Higher Education (grant no. N N518 385237) and the European Social Fund implemented under the Human Capital Operational Programme (POKL), project: "Preparation and Realization of Medical Physics Specialty". The researchers from the Gliwice Oncology Centre, Poland (Prof. M. Sokół, Prof. B. Billewicz, Dr. B. Niewiadomska, M.Sc. A. Skorupa), are greatly appreciated for co-operation and MRI scanning of a PABIG $^{\text {nx }}$ vial.

\section{References}

[1] J. Adamovics, M. Maryanski, Rad. Prot. Dosimetry 120, 107 (2006).

[2] J.C. Gore, Y.S. Kang, R.J. Schultz, Phys. Med. Biol. 29, 1189 (1984a).

[3] J.C. Gore, Y.S. Kang, R.J. Schultz, Magn. Reson. Imaging 2, 244 (1984b).

[4] L.E. Olsson, S. Petersson, L. Ahlgren, Phys. Med. Biol. 34, 43 (1989). 
[5] R.J. Schulz, A.E. de Guzman, D.E. Nguten, J.C. Gore, Phys. Med. Biol. 35, 1611 (1990).

[6] T. Kron, P. Metealfe, J.M. Pope, Phys. Med. Biol. 38, 139 (1993).

[7] C. Duzenli, R. Sloboda, D. Robinson, Phys. Med. Biol. 39, 1577 (1994).

[8] G. Bartesaghi, M. Carrera, G. Gambarini, A. Negri, J. Phys.: Conf. Ser. 164, 012063 (2009).

[9] H. Fricke, S. Morse, Am. J. Roent. Rad. Ther. 18, 430 (1927).

[10] M.J. Maryanski, J.C. Gore, R.P. Kennan, R.J. Schulz, Magn. Reson. Imaging 11, 253 (1993).

[11] M.J. Maryanski, R.J. Schultz, G.S. Ibbott, J.C. Gatenby, J. Xie, D. Horton, J.C. Gore, Phys. Med. Biol. 39, 1437 (1994).

[12] M.J. Maryanski, G.S. Ibbott, P. Eastman, R.J. Schultz, J.C. Gore, Med. Phys. 23, 699 (1996).

[13] P.M. Fong, D.C. Keil, M.D. Does, J.C. Gore, Phys. Med. Biol. 46, 3105 (2001).

[14] Y. de Deene, C. Hurley, A.J. Venning, K. Vergote, M. Mather, B.J. Healy, C. Boldock, Phys. Med. Biol. 47, 3441 (2002).

[15] C. Hurley, A. Venning, C. Baldock, Appl. Radiat. Isot. 63, 443 (2005).

[16] A. Venning, B. Healy, K. Nitschke, C. Baldock, Nucl. Instrum. Methods Phys. Res. A 555, 396 (2005a).

[17] A. Venning, B. Hill, S. Brindha, B. Healy, C. Baldock, Phys. Med. Biol. 50, 3875 (2005b).

[18] E. Pappas, T. Maris, A. Angelopoulos, M. Paparigopoulou, L. Sakelliou, P. Sandilos, S. Voyiatzi, L. Vlachos, Phys. Med. Biol. 44, 2677 (1999).
[19] M. Kozicki, PhD thesis. Institute of Applied Radiation Chemistry, Technical University of Łódź, Poland 2004.

[20] M. Kozicki, L. Petrokokkinos, P. Papagiannis, L. Sakelliou, A. Angelopoulos, E. Pappas, J.M. Rosiak, Biomed. Tech. 50, 1368 (2005).

[21] M. Kozicki, P. Maras, K. Rybka, T. Bieganski, S. Kadłubowski, L. Petrokokkinos, Macromol. Symp. 254, 345 (2007).

[22] E. Pantelis, C. Antypas, L. Petrokokkinos, P. Karaiskos, P. Papagiannis, M. Kozicki, E. Georgiou, L. Sakelliou, I. Seimenis, Med. Phys. 35, 2312 (2008).

[23] A. Moutsatsos, P. Karaiskos, L. Petrokokkinos, K. Zourari, E. Pantelis, L. Sakelliou, I. Seimenis, C. Constantinou, A. Peraticou, E. Georgiou, J. Phys.: Conf. Ser. 250, 012060 (2010).

[24] A.E. Papadakis, T.G. Maris, F. Zacharopoulou, E. Pappas, G. Zacharakis, J. Damilakis, Phys. Med. Biol. 52, 5069 (2007).

[25] K. Gut, D. Nabaglo, Acta Phys. Pol. A 116, 307 (2009).

[26] M. Kozicki, J.M. Rosiak, in: Proc. Second Int. Symp. on Reactive Polymers in Inhomogeneous Systems, in Melts and at Interfaces, Dresden (Germany), Book of abstract, 2003, S3/33.

[27] M. Kozicki, Rad. Phys. Chem. 80, 1419 (2011). 